\title{
EL GÉNERO Y SU INCIDENCIA EN LOS EMPRENDIMIENTOS DE LOS GRADUADOS EN MARKETING. ULEAM, 2015-2019
}

\author{
Ing. Washington Lenin Valencia Vásquez \\ Universidad Laica "Eloy Alfaro" de Manabí \\ ORCID: https://orcid.org/0000-0001-5639-4246 \\ lenin.valencia2014@gmail.com \\ Ing. Deodato Loor Chávez PhD. \\ Universidad Laica Eloy Alfaro de Manabí \\ ORCID: https://orcid.org/0000-0001-8123-4543 \\ deodato.loor@uleam.edu.ec \\ Ing. Priscella María Ponce Valdez \\ Universidad Laica "Eloy Alfaro" de Manabí \\ ORCID: https://orcid.org/0000-0003-0399-9836 \\ priscellaponce4@gmail.com \\ Ing. Dayni Lisset Palacios Molina., PhD \\ Universidad Laica "Eloy Alfaro" de Manabí \\ ORCID: https://orcid.org/0000-0003-2201-2507 \\ dayni.palacios@uleam.edu.ec
}

Para citar este artículo puede utilizar el siguiente formato:

Washington Lenin Valencia Vásquez, Deodato Loor Chávez, Priscella María Ponce Valdez y Dayni Lisset Palacios Molina: "El género y su incidencia en los emprendimientos de los graduados en marketing. ULEAM, 2015-2019", Revista Caribeña de Ciencias Sociales (vol 10, № 8 octubrediciembre 2021, pp. 144-158. En línea:

https://doi.org/10.51896/caribe/SEAL6731

\section{RESUMEN}

El género desde hace mucho tiempo ha sido un factor predominante para desarrollar algún tipo de actividad económica, es así que la actividad de emprender no es la excepción, debido a que el emprendimiento tanto del hombre como de la mujer se encuentra condicionado de acuerdo a la manera en cómo se configure cada actividad económica y como la personalidad de ambos sujetos varía en función de su género, modificando la forma en la que cada individuo se desenvuelve en la administración de un negocio. El presente trabajo de investigación tuvo como objetivo determinar de qué manera el género incide en los emprendimientos de los graduados de la carrera de Marketing de la Universidad Laica "Eloy Alfaro" de Manabí periodo 2015-2019. El estudio conto con una metodología cuali-cuantitativa, con la aplicación del método inductivo y deductivo, junto a un diseño exploratorio, descriptivo, explicativo, correlacional, transversal, no experimental, bibliográfico. Mediante el programa SPSS 25, con el coeficiente de correlación p (Rho) de Spearman, se pudo 
comprobar la hipótesis general y específicas, demostrando que tanto el género como los factores psicosociales, sociológicos, socioeconómicos y modelo de negocio inciden en los emprendimientos de los graduados de la carrera de Marketing de la Universidad Laica "Eloy Alfaro" de Manabí periodo 2015-2019, alcanzando respectivamente los siguientes coeficientes de $(0,982),(0.993),(0,958)$, $(0,982)$ y $(0,958)$, significando que se validan positiva y significativamente.

Palabras Claves: Género, Emprendimiento, Factor Psicosocial, Factor Sociológico, Factor socioeconómico, Modelo de negocio.

\section{GENDER AND ITS IMPACT ON THE ENTREPRENEURSHIP OF MARKETING GRADUATES. ULEAM, 2015-2019}

\section{ABSTRACT}

Gender has long been a predominant factor to develop some type of economic activity, so the activity of entrepreneurship is not the exception, because the entrepreneurship of both men and women is conditioned according to the The way in which each economic activity is configured and how the personality of both subjects varied according to their gender, modifying the way in which each individual develops in the administration of a business. The main objective of this research is to determine how gender affects the entrepreneurship of graduates of University Laica "Eloy Alfaro" de Manabí period 2015-2019. The study had a qualitative-quantitative methodology, with the application of the inductive and deductive method, together with an exploratory, descriptive, explanatory, correlational, cross-sectional, non-experimental, bibliographic design. Using the SPSS 25 program, with Spearman's correlation coefficient $p$ (Rho), it was possible to verify the general and specific hypotheses, demonstrating that both gender and psychosocial, sociological, socioeconomic and business model factors affect the enterprises of the companies. graduates of the Marketing career of the Lay University "Eloy Alfaro" of Manabí period 2015-2019, respectively reaching the following coefficients of (0.982), (0.993), (0.958), (0.982) and (0.958), meaning that they are positively and significantly validated.

Key Words: Gender, Entrepreneurship, Psychosocial Factor, Sociological Factor, Socio-economic Factor, Business Model.

\section{INTRODUCCIÓN}

En la actualidad gran parte del desarrollo económico y social de los países se ve determinado por la iniciativa que tiene su población por emprender, empujados por diversos factores como la necesidad económica, las oportunidades de negocio y el deseo de lograr independencia y estabilidad económica, pero indistintamente de la necesidad que tenga cada persona, existe un factor determinante que estimula o aplaca esta iniciativa, este es el rol que ha desempeñado en la población, cada individuo según su género.

Históricamente, las actividades económicas o las relaciones laborales estaban determinadas sobre la base de un modelo familiar en donde el padre era aquel que proveía y la madre la 
encargada del hogar, dando como resultado una división sexual del trabajo manifiestamente establecida: en donde cada individuo cumplía un rol determinado en la sociedad, favoreciendo visiblemente al hombre como agente productivo, en cuanto por norma debía proveer la subsistencia familiar con su trabajo como cabeza de familia, mientras que la mujer asumía un rol cuidador y reproductivo, sin remuneración, dedicado al parto, la crianza, las tareas del hogar y el cuidado de los menores, enfermos y dependientes.

Hasta hace unas décadas, las responsabilidades y roles inherentes a estos ámbitos eran efectuados por hombres y mujeres de forma diferenciada debido a la tradicional división de roles por sexos. No obstante, los cambios sociales, políticos e ideológicos han llevado a la mujer a la búsqueda de igualdad en relación con los hombres, iniciando con su inserción en el campo laboral, sin embargo, sigue habiendo una brecha significativa con el sexo masculino en términos de desempleo, esta temática puede ser impulsada debido a que las mujeres tienden a no incursionar en profesiones que requieran trabajos físicamente forzosos o no suele ser normal la contratación de las mismas en ellos, por otra parte hombres y mujeres por el simple hecho de poseer características diferenciadas efectúan trabajos enfocándose en aspectos distintos al realizarlos, es por esto que la personalidad de acuerdo al género que se posee definen como estas llevan a cabo un emprendimiento, en que aplican mayor importancia, que habilidades y competencias desarrollan más y que obstáculos son aquellos que resaltan en función del sexo.

En el año 2019, en Ecuador se ven diferencias en las percepciones individuales entre hombres y mujeres, mismas que se han mantenido aproximadamente desde el 2008, siendo estadísticamente significativas. Específicamente, en promedio en el período 2008-2019, más hombres (52.93\%) que mujeres (49.99\%) percibieron oportunidades para emprender; así mismo, más hombres $(76.56 \%)$ que mujeres consideran que cuentan con capacidades para iniciar un negocio que las mujeres (69.68\%). Finalmente, las mujeres (38.54\%) mostraron que el miedo al fracaso constituye una barrera para iniciar un negocio en mayor proporción que los hombres (30.91\%). A si mismo existen diferencias de género, donde las mujeres emprenden en mayor proporción para ganarse la vida ante la escasez de empleo, y en menor proporción para hacer la diferencia o acumular riqueza, además la tradición familiar es un motivo más común entre las mujeres. (GEM, 2019-2020, págs. 23,29)

En Ecuador, la actividad emprendedora está creciendo con más fuerza y cada vez se incrementa el número de emprendedores. Según los datos estadísticos del Global Entrepreneurship Monitor (2019/2020), Ecuador mantiene una actividad emprendedora predominante entre los países de América Latina y El Caribe con una participación del 33.6\%, ocupando el primer lugar. Ecuador es uno de los países con mayor paridad de género en cuanto a la TEA. Sin embargo, a partir de 2015 las proporciones de hombres y mujeres en la TEA se han venido alejando ligeramente de la paridad. En 2019, el total de emprendedores TEA estuvo compuesto en un $53.5 \%$ por hombres y $46.5 \%$ por mujeres. La proporción de hombres con negocios nuevos (52.30\%) continúa siendo superior a la de las mujeres, manteniendo la tendencia en los últimos años. Para los negocios establecidos, se observa una mayor proporción de hombres $(62 \%)$, que muestra una marcada diferencia con las mujeres propietarias (38.0\%). En otras palabras, a pesar de que una cantidad similar de hombres y 
mujeres emprenden, una proporción mayor de hombres lograría sostener sus negocios por más de 42 meses. Cabe resaltar que las disparidades de género vienen desde antes de la decisión de emprender. Tanto los resultados del presente reporte, como los de años pasados, muestran que los hombres manifiestan una mayor autopercepción de capacidades para emprender, y menor temor al fracaso como barrera para iniciar un negocio. (ESPAE, 2020, pág. 25)

La universidad es aquella entidad encargada de guiar a sus alumnos hacia la contribución con la economía de un país y es por esto que la Universidad Laica "Eloy Alfaro" de Manabí debe ser gestora de una cultura emprendedora, más aún si hablamos de la facultad ciencias administrativas, específicamente de la carrera de marketing/mercadotecnia, donde se desarrolla el presente estudio, la influencia de la formación académica debería estar íntegramente relacionada a la intención emprendedora de los estudiantes de la carrera, desafortunadamente esta no es fomentada adecuadamente, dado que la escuela no identifica las actitudes de ambos géneros para integrarlos en una metodología de aprendizaje que incentive a los futuros graduados a emprender, tampoco orientan a los alumnos a informarse sobre el entorno y la demanda insatisfecha que existe en el mismo, encontrando así oportunidades reales acorde al tipo de industria, contribuyendo con un modelo de negocio que la dinamice y que a través de sus conocimientos en mercadotecnia pueda aportar a su expansión.

Debido a esto la problemática se centra en descubrir y describir de qué manera el género incide en los emprendimientos de los graduados de la carrera de marketing de la Universidad Laica "Eloy Alfaro" de Manabí periodo 2015-2019, evidenciando las presuntas razones que contribuyen a que esta correlación de variables sea posible, partiendo de la conjetura de que las mujeres y los hombres emprenden ideas de negocios en función de su género, más que en la demanda real insatisfecha del mercado, puesto que el género se comprende de estereotipos y roles de género, estos a su vez moldean a la personalidad, la cual condiciona a que tanto hombres como mujeres realicen las mismas acciones pero con diferentes enfoques, por lo que los negocios 0 emprendimientos estarían dictaminados por características de la personalidad propias de cada sexo.

\section{MARCO TEÓRICO}

\section{Género}

El género es el "conjunto de creencias, rasgos personales, actitudes, sentimientos, valores, conductas y actividades que diferencian a hombres y mujeres a través de un proceso de construcción social que tiene varias características, mismo que se construye socialmente y hace referencia a lo que se considera femenino o masculino, tratándose de una concepción susceptible de variar según el lugar y el momento" (Venegas, 2011) citado en Morán y Macías (2016, pág. 39)

Los argumentos planteados por los diferentes actores concuerdan que el género es una construcción de los aspectos sociales, basados en las funciones, comportamiento, actividades y atributos que cada sociedad considera apropiados para hombres y mujeres, estableciendo así semejanzas y diferencias entre ellos. 
El género "es una categoría construida para explicar las diferencias sociales que hay entre hombres y mujeres, para explicar las causas históricas de la desigualdad en sus relaciones y la subordinación de las mujeres, por tanto, género se refiere a las diferencias psicológicas, sociales y culturales que hay entre mujeres y hombres". (Poma y Mendoza, 2012) citado en Morán y Macías (2016, pág.39)

El género es la construcción social y cultural de las diferencias sexuales, es decir, es el elemento por medio del cual se crea esta idea de la dicotomía y complementariedad entre los sexos varón/mujer. El género como sistema reproduce estas ideas mediante los procesos de socialización que tienen lugar en instituciones sociales como el matrimonio, la familia, la escuela, el trabajo, etc., y las convierte en prácticas mediante la asignación diferenciada y desigual de roles y la instauración de pautas de comportamiento que representan un deber ser para los hombres y otro para las mujeres y que desconoce otras identidades de sexo y de género. (Defensoría del Pueblo, 2016) citado en Massuh, (2018, pág.29)

\section{El papel de los estereotipos en el desarrollo profesional}

La Teoría del Rol Social de Género informa de cómo los roles, dentro de una estructura social determinada, influyen en las relaciones entre grupos, así como del vínculo existente entre dichos roles y los estereotipos de género. Mediante normas se asignan responsabilidades y roles a los miembros de la sociedad y se garantizan su funcionamiento, se produce una segmentación laboral que se traduce en una segregación sexual del mercado de trabajo (García-Leiva, 2005).

Además, según (Cejka \& Eagly, 1999), la Teoría del Rol Social manifiesta que existen vínculos recíprocos entre la segregación sexual de las ocupaciones y los estereotipos de género. Por una parte, la distribución de los individuos en las diversas ocupaciones de acuerdo con la segregación sexual apuntala la existencia de estereotipos de género puesto que, ya sea por observación directa o bien indirecta (medios de comunicación), los hombres y las mujeres se exponen ante la sociedad mostrándose tanto en las esferas públicas como privadas. La relación de causalidad es recíproca puesto que el arraigo de los estereotipos de género en las mentalidades provoca que los individuos conciban ocupaciones apropiadas para cada sexo, hasta el punto de que aquellas personas que trabajan en ocupaciones incongruentes al género, son víctimas de un conflicto de rol, especialmente cuando es el caso de mujeres que se ocupan en trabajos típicos de hombres.

Para (Cejka \& Eagly, 1999), los estereotipos de género promueven y reflejan la segregación sexual en el mercado de trabajo hasta el extremo de que los trabajos que conllevan poder, salarios altos, prestigio y autoridad a nivel social son, generalmente, estereotipados como masculinos. Asimismo, (Watson \& Newby, 2005), manifiestan que a partir de la década de los 70 , en las sociedades occidentales, se empezó a incorporar masivamente la mujer al mercado de trabajo junto con la mejora de sus niveles de formación, conducido a que, progresivamente, las barreras hayan cambiado de lugar, de modo que las mujeres han iniciado un proceso de incorporación sin retorno a empleos tradicionalmente masculinos, mientras que los hombres también lo han hecho en otros 
femeninos, asumiendo paulatinamente parte de las tareas domésticas diarias e incluyendo más tiempo al cuidado de los hijos.

Sin embargo, si se estudian las imágenes de genero se puede observar como la tradicional concepción de mujer (más amable, cariñosa y comprensiva que el hombre) no se mantiene si se describe a una mujer trabajadora en lugar de a una ama de casa (Eagly \& Steffen, 1984).

Por otro lado, (Eagly \& Wood, 1982), mencionan que la ocupación de un puesto de alta responsabilidad en el mundo laboral les hace ser vistas tan independientes como los varones, mientras que cuando son subordinadas se las considera más sumisas. Sin embargo, mediante el mismo proceso, a los hombres que desempeñan las tareas del hogar se les suponen tan sensibles como a las mujeres en las relaciones interpersonales, creencia que no se sostiene si es otra la ocupación. Así también (Gómez Esteban, 2000), considera que el prejuicio aparece cuando las mujeres contradicen los estereotipos prescriptivos y ocupan con éxito cargos tradicionalmente masculinos, aun tienden a ser penalizadas con rechazo social.

Más concretamente, en el ámbito de las organizaciones, las investigaciones demuestran la prevalencia de los estereotipos de género, hasta el punto de que la alta dirección se considera un negocio de hombres, mientras que las tareas administrativas como los puestos de secretarias, son un trabajo de mujeres, (Cacouault, 2000).

Para la historiadora económica (Gálvez, 2004), el oficio de manager es uno de los más ilustrativos en el estudio de la construcción social de las profesiones desde la perspectiva de género: a pesar de que las mujeres puedan estar en igualdad de condiciones que los hombres en cuanto a capacitación y formación, las ejecutivas siguen siendo minoritarias en la profesión pues la imagen del gestor coincide con atributos que se consideran masculinos. Dicha imagen se caracteriza por el principio de autoridad y la capacidad para tomar decisiones. Además, hay una diferencia en el reparto de usos del tiempo entre sexos: se da por supuesto que la alta jerarquía empresarial requiere de una larga jornada laboral para ser ejercida, lo que constituye una barrera de entrada para las mujeres, que se encuentran ante la disyuntiva de tener que elegir entre la vida profesional y la familiar.

Para (Schein, 1975), el desarrollo de la carrera profesional de las mujeres como gestoras se ve obstaculizado por los estereotipos de que son objeto (falta de ambición, inseguridad, incapacidad para tomar decisiones y asumir riesgos, emotividad), los cuales concluyen en la no idoneidad para mandar puesto que se trata de una habilidad que no se puede aprender, sino que es natural en los hombres y no en las mujeres, es así que el pensamiento de que el manager debe ser masculino es algo que ha prevalecido como una verdad evidente.

\section{Hipótesis General}

El género incide en los emprendimientos de los graduados de la carrera de Marketing de la Universidad Laica "Eloy Alfaro" de Manabí periodo 2015-2019. 


\section{Hipótesis Específicas}

Los factores psicosociales inciden en los factores socioeconómicos de los emprendimientos de los graduados de la carrera de Marketing de la Universidad Laica "Eloy Alfaro" de Manabí periodo 2015-2019.

Los factores psicosociales inciden en el modelo de negocio de los emprendimientos de los graduados de la carrera de Marketing de la Universidad Laica "Eloy Alfaro" de Manabí periodo 20152019.

Los factores sociológicos inciden en los factores socioeconómicos de los emprendimientos de los graduados de la carrera de Marketing de la Universidad Laica "Eloy Alfaro" de Manabí periodo 2015-2019.

Los factores sociológicos inciden en el modelo de negocio de los emprendimientos de los graduados de la carrera de Marketing de la Universidad Laica "Eloy Alfaro" de Manabí periodo 20152019.

\section{METODOLOGÍA}

En el presente estudio se utilizó tanto el método inductivo como el deductivo, el primero consta de investigaciones cualitativas que se basan más en una lógica y proceso inductivo (explorar y describir, y luego generar perspectivas teóricas). Van de lo particular a lo general, mientras que el deductivo consta de investigaciones cuantitativas que se valen de la lógica, iniciando con la teoría, y de ésta se derivan expresiones lógicas denominadas hipótesis que el investigador somete a prueba (Hernández, Fernández, \& Baptista, 2014, pág. 6-8).

De la misma manera, se utilizó la investigación cuantitativa, cualitativa, exploratoria, descriptiva, explicativa, correlacional, transversal y bibliográfica, determinada en las variables y dimensiones de la investigación, para demostrar las correlaciones se empleó el programa estadístico SPSS versión 25.

\section{Diseño de investigación}

Se desarrolló un instrumento de medición en escala de Likert, de tipo no experimental. De acuerdo con Hernández, Fernández, \& Baptista (2014) manifiestan que los mismos "son estudios realizados sin la manipulación deliberada de variables y en los que sólo se observan los fenómenos en su ambiente natural para analizarlos" (pág. 152). La encuesta permitió conocer con mayor certeza de qué manera el género incide en los emprendimientos de los graduados de la carrera de Marketing de la Universidad Laica "Eloy Alfaro" de Manabí periodo 2015-2019.

\section{Unidad de análisis}

Se toma como unidad de análisis a la población de 334 graduados de la carrera de Marketing en la matriz (Manta) de la Universidad Laica "Eloy Alfaro" de Manabí que tuvieron o actualmente cuentan con un emprendimiento, información adquirida de una base de datos creada a partir de la 
información obtenida por la secretaría general de la ULEAM, correos, redes sociales y registros del Servicio de Rentas Internas de los sujetos estudiados.

\section{Población de estudio}

Según Santiesteban (2014), la muestra es un conjunto extraído por un procedimiento técnico de la población. Es un grupo relativamente pequeño, es un subgrupo de esta que va a ser estudiada y sobre la cual se pretende generalizar los resultados, constituyen de unidades de análisis, que supuestamente representan en mayor o menor grado las características de la población. (pág. 262).

Se empleó la fórmula para una población finita a los 334 graduados en Marketing en la Universidad Laica "Eloy Alfaro" de Manabí durante el periodo 2015-2019 en la matriz en Manta. En la tabla 1 , se detallan los datos de los graduados con emprendimientos activos y suspendidos.

\section{Tabla 1:}

Datos de Graduados con emprendimientos activos y suspendidos

Dimensión de la muestra

\begin{tabular}{|c|c|c|c|c|c|c|c|c|c|c|c|c|c|c|c|}
\hline \multirow{3}{*}{ AÑOS } & \multicolumn{4}{|c|}{ COMERCIO } & \multirow{3}{*}{ TOTAL } & \multicolumn{4}{|c|}{ SERVICIO } & \multirow{3}{*}{ TOTAL } & \multicolumn{4}{|c|}{ PRODUCCIÓN } & \multirow{3}{*}{ TOTAL } \\
\hline & \multicolumn{2}{|c|}{ ACTIVO } & \multicolumn{2}{|c|}{ SUSPENDIDO } & & \multicolumn{2}{|c|}{ ACTIVO } & \multicolumn{2}{|c|}{ SUSPENDIDO } & & \multicolumn{2}{|c|}{ ACTIVO } & \multicolumn{2}{|c|}{ SUSPENDIDO } & \\
\hline & $\mathbf{H}$ & M & H & $\mathbf{M}$ & & $\mathbf{H}$ & $\mathbf{M}$ & H & $\mathbf{M}$ & & $\mathbf{H}$ & $\mathbf{M}$ & H & $\mathbf{M}$ & \\
\hline 2015 & 5 & 17 & 5 & 12 & 39 & 7 & 11 & 9 & 15 & 42 & 1 & 1 & 0 & 1 & 3 \\
\hline 2016 & 11 & 22 & 8 & 13 & 54 & 15 & 26 & 17 & 18 & 76 & 1 & 1 & 1 & 0 & 3 \\
\hline 2017 & 0 & 5 & 3 & 3 & 11 & 5 & 10 & 7 & 6 & 28 & 0 & 1 & 0 & 0 & 1 \\
\hline 2018 & 8 & 12 & 1 & 8 & 29 & 10 & 7 & 4 & 8 & 29 & 0 & 2 & 0 & 0 & 2 \\
\hline \multirow[t]{2}{*}{2019} & 4 & 5 & 2 & 0 & 11 & 2 & 1 & 0 & 1 & 4 & 1 & 1 & 0 & 0 & 2 \\
\hline & & & & & 144 & & & & & 179 & & & & & 11 \\
\hline \multicolumn{16}{|c|}{ TOTAL DE EMPRENDIMIENTOS = 334} \\
\hline
\end{tabular}

Fuente: Elaboración de autores

Acorde a las características de la investigación fue preciso utilizar el muestreo probabilístico aleatorio simple, considerando el margen de error y el nivel de confianza. Se empleó la siguiente fórmula para calcular la muestra, donde: $P=50 \%$ (probabilidad de que ocurra el evento), $Q: 50 \%$ (probabilidad de que no ocurra el evento), Z2: 1,96(nivel de confianza), N: población (940), $E^{2}$ : 0,05(Margen de error), N: ¿? (Tamaño de la muestra).

$$
\begin{aligned}
& n=\frac{Z^{2} P Q N}{E^{2}(\mathrm{~N}-1)+Z^{2} P Q} \\
& n=\frac{z 2 * N p q}{e 2(N-1)+Z 2 p q} \\
& n=\frac{3,8416 * 334 * 0,5 * 0,5}{0,0025(334-1)+3,8416 * 0,5 * 0,5} \\
& n=\frac{320,7736}{1,7929} \\
& n=179
\end{aligned}
$$


De acuerdo con los resultados obtenidos después de utilizar la formula, el tamaño de la muestra es de 179 graduados a encuestar.

\section{Recolección de datos}

A través de una encuesta conformada por 30 preguntas, estructurada en escala de Likert y aplicada a 179 graduados, se logró recolectar los datos necesarios para cumplir con el objetivo de la investigación. El instrumento fue validado por expertos, en el que se analizó la validez de contenido, criterio y constructo, así como también por medio de programa estadístico SPSS 25.

\section{RESULTADOS}

Mediante la aplicación del programa estadístico SPSS 25 , se procedió la utilización del coeficiente Alfa de Cronbach para establecer la fiabilidad del instrumento, con el cual, de acuerdo al baremo estadístico clásico, se demuestra una alta confiabilidad, con un valor de 0,992. La tabla 2 , describe el análisis de fiabilidad del instrumento utilizado para el estudio.

Tabla2:

Análisis de fiabilidad

\begin{tabular}{rrr} 
& $\begin{array}{c}\text { Estadísticas de fiabilidad } \\
\text { Alfa de Cronbach } \\
\text { basada en elementos } \\
\text { estandarizados }\end{array}$ & \multicolumn{2}{|c}{ N de elementos } \\
\hline Alfa de Cronbach &, 993 & 30 \\
\hline, 992 &
\end{tabular}

Recurso: Programa estadístico SPSS 25

\section{Comprobación de Hipótesis}

Empleando el sistema estadístico SPSS-25, se efectuó las pruebas de hipótesis, a través coeficiente $p$ "Rho" de Spearman; en la investigación, de corte longitudinal, se comprobó la correlación entre las variables "Género y emprendimiento", como se muestra en la tabla 4, con los siguientes resultados:

\section{Tabla 3:}

Resultados de prueba de hipótesis

\begin{tabular}{|c|c|c|c|c|c|}
\hline Hipótesis & $\begin{array}{c}\text { Variable } \\
\text { Independiente }\end{array}$ & $\begin{array}{c}\text { Variable } \\
\text { Dependiente }\end{array}$ & Sig & $\begin{array}{c}\text { Rho de } \\
\text { Spearman }\end{array}$ & Resultado \\
\hline H. General & X. Género & $\begin{array}{c}\text { Y. } \\
\text { Emprendimiento }\end{array}$ & ,000 & ,982 & Se rechaza Ho \\
\hline H. Específica 1 & $\begin{array}{l}\text { X1. Factores } \\
\text { psicosociales }\end{array}$ & $\begin{array}{c}\text { Y1. Factores } \\
\text { socioeconómicos }\end{array}$ & ,000 & ,993 & Se rechaza Ho \\
\hline H. Específica 2 & $\begin{array}{l}\text { X2. Factores } \\
\text { psicosociales }\end{array}$ & $\begin{array}{l}\text { Y2. Modelo de } \\
\text { negocio }\end{array}$ & ,000 & ,958 & Se rechaza Ho \\
\hline H. Específica 3 & $\begin{array}{l}\text { X3. Factores } \\
\text { sociológicos }\end{array}$ & $\begin{array}{c}\text { Y3. Factores } \\
\text { socioeconómicos }\end{array}$ & ,000 & 982 & Se rechaza Ho \\
\hline H. Específica 4 & $\begin{array}{l}\text { X4. Factores } \\
\text { sociológicos }\end{array}$ & $\begin{array}{l}\text { Y4. Modelo de } \\
\text { negocio }\end{array}$ & ,000 & 943 & Se rechaza Ho \\
\hline
\end{tabular}


${ }^{* *}$ La correlación es significativa en el nivel de 0,01 (bilateral)

Recurso: Programa estadístico SPSS 25.

Hipótesis General $(\mathrm{X})$

H0: El género NO inciden en los emprendimientos de los graduados de la carrera de Marketing de la Universidad Laica "Eloy Alfaro" de Manabí periodo 2015-2019.

H1: El género SI inciden en los emprendimientos de los graduados de la carrera de Marketing de la Universidad Laica "Eloy Alfaro" de Manabí periodo 2015-2019.

\section{Análisis de prueba}

La validación de la hipótesis general $(\mathrm{X})$, procesados los datos, nos muestra una significación de 0,00 y un coeficiente $p$ (Rho) Spearman de 0,982, afirmándose la hipótesis, lo cual evidencia un nivel de correlación "positiva perfecta" en el cruce de variables.

\section{Hipótesis específica 1 (X1)}

H0: Los factores Psicosociales NO inciden en los factores socioeconómicos de los emprendimientos de los graduados de la carrera de Marketing de la Universidad Laica "Eloy Alfaro" de Manabí periodo 2015-2019.

$\mathrm{H} 1$ : Los factores psicosociales $\mathrm{SI}$ inciden en los factores socioeconómicos de los emprendimientos de los graduados de la carrera de Marketing de la Universidad Laica "Eloy Alfaro" de Manabí periodo 2015-2019.

\section{Análisis de prueba}

El procesamiento de datos en el SPSS 25 para la hipótesis específica (X1) establece una significación bilateral de 0,00 con un coeficiente de $p$ (Rho) de Spearman de 0,993, ratificando positivamente el supuesto, tal como lo demuestra el nivel de correlación.

\section{Hipótesis específica 2 (X2)}

HO: Los factores psicosociales NO inciden en el modelo de negocio de los emprendimientos de los graduados de la carrera de Marketing de la Universidad Laica "Eloy Alfaro" de Manabí periodo 2015-2019.

$\mathrm{H} 1$ : Los factores psicosociales SI inciden en el modelo de negocio de los emprendimientos de los graduados de la carrera de Marketing de la Universidad Laica "Eloy Alfaro" de Manabí periodo 2015-2019. 


\section{Análisis de prueba}

El procesamiento de datos en el SPSS 25 para la hipótesis específica (X2) determina una significación bilateral de 0,00 con un coeficiente de $p$ (Rho) de Spearman de 0,958, ratificando positivamente el supuesto, tal como lo demuestra el nivel de correlación.

\section{Hipótesis específica 3 (X3)}

$\mathrm{HO}$ : Los factores sociológicos $\mathrm{NO}$ inciden en los factores socioeconómicos de los emprendimientos de los graduados de la carrera de Marketing de la Universidad Laica "Eloy Alfaro" de Manabí periodo 2015-2019.

$\mathrm{H} 1$ : Los factores sociológicos $\mathrm{SI}$ incide en los factores socioeconómicos de los emprendimientos de los graduados de la carrera de Marketing de la Universidad Laica "Eloy Alfaro" de Manabí periodo 2015-2019.

\section{Análisis de prueba}

El procesamiento de datos en el SPSS 25 para la hipótesis específica (X3) determina una significación bilateral de 0,00 con un coeficiente de $p$ (Rho) de Spearman de 0,982, ratificando positivamente el supuesto, tal como lo demuestra el nivel de correlación.

\section{Hipótesis específica 4 (X4)}

H0: Los factores sociológicos NO inciden en el modelo de negocio de los emprendimientos de los graduados de la carrera de Marketing de la Universidad Laica "Eloy Alfaro" de Manabí periodo 2015-2019.

$\mathrm{H1}$ : Los factores sociológicos SI incide en el modelo de negocio de los emprendimientos de los graduados de la carrera de Marketing de la Universidad Laica "Eloy Alfaro" de Manabí periodo 20152019.

\section{Análisis de prueba}

El procesamiento de datos en el SPSS 25 para la hipótesis específica (X4) determina una significación bilateral de 0,00 con un coeficiente de $p$ (Rho) de Spearman de 0,943, ratificando positivamente el supuesto, tal como lo demuestra el nivel de correlación.

\section{DISCUSIÓN}

En las últimas décadas se observa un considerable incremento de la cantidad de mujeres que emprenden algún negocio. Sin embargo, aún no existe un equilibrio entre la proporción de mujeres que emprenden frente a los hombres que realizan esta misma acción. El emprendimiento se ha ubicado como una de las principales estrategias, no solo de inserción laboral, sino también de lucha contra la pobreza, es así que ante esta realidad actualmente hasta los centros de enseñanza de todo nivel tienen cátedras sobre emprendimiento. 
La mujer ha ganado terreno en espacios de poder políticos, económicos, profesionales, laborales, personales, no obstante, la distribución de riqueza entre género aún está lejos de llegar a la equidad, es así que, en el ámbito emprendedor, en los últimos años, la participación del género femenino ha tenido un evidente aumento, aunque en la mayoría de países la brecha de género persiste (Kelley, Brush, Greene, \& Litovsky., 2011).

La imagen del emprendedor siempre ha estado ligada con la identidad o rasgos masculinos, por lo que, bajo dicha caracterización, se cree que hay una incompatibilidad entre sexo femenino y el emprendimiento. Esta teoría de la amenaza del estereotipo de género justifica esta situación debido al débil involucramiento de la mujer en la creación de empresas. (Steele, 1998)

Asimismo, se cree que algunos rasgos característicos de las mujeres, como la adaptabilidad, la orientación hacia los demás, la amabilidad, la humildad, la flexibilidad, entre otros., son aspectos positivos que pueden encaminar a realizar emprendimientos, generando así un cambio de paradigma en el ámbito de la creación de empresas. Esta redefinición del involucramiento de la mujer en los negocios rompe el distanciamiento que históricamente ha existido con la esfera productiva, en tal sentido, la influencia de los estereotipos de género en el emprendimiento debe ser analizado desde una óptica más amplia y neutral. Lo que permite aseverar que todos, mujeres y hombres, somos agentes activos en el proceso de socialización, en cualquier ámbito de nuestras vidas, ya que los seres humanos somos cómplices de la construcción social. (Gálvez, 2004).

\section{CONCLUSIONES}

Para concluir se puede afirmar que la hipótesis general se cumple a cabalidad, al comprobar correlacionalmente la incidencia que tiene el género en los emprendimientos de los graduados de la carrera de Marketing de la Universidad Laica "Eloy Alfaro" de Manabí periodo 2015-2019; así como las específicas a través de la comprobación de correlación con las dimensiones Factores psicosociales, factores sociológicos, factores socioeconómicos y modelo de negocio.

Con el programa estadístico SPSS, se validó la hipótesis general, resultados que evidencian la significancia correlacional de las variables, con un $p$ (Rho) de Spearman de 0,982. Lo que quiere decir que el género en los emprendimientos de los graduados de la carrera de Marketing de la Universidad Laica "Eloy Alfaro" de Manabí periodo 2015-2019, por ello se debería efectuar un plan de capacitación en identificación de oportunidades de negocio para el alumnado y graduados de la carrera de marketing/mercadotecnia de la Universidad Laica "Eloy Alfaro" de Manabí, puesto que así se podrán incentivar ideas de negocios en el alumnado más cercanas a la demanda real insatisfecha del mercado, fortalecer conocimientos y orientar sobre el título universitario en el campo laboral.

Se pudo además validar la hipótesis específica 1, con un p (Rho) de Spearman de 0,993, articulando que los factores psicosociales inciden en los factores socioeconómicos de los emprendimientos de los graduados de la carrera de Marketing de la Universidad Laica "Eloy Alfaro" de Manabí periodo 2015-2019, por ello el gobierno debería garantizar la existencia de políticas públicas de fomento al emprendimiento femenino, para que las mujeres tengan mayor acceso a 
financiamiento, debido a que en la actualidad las instituciones bancarias las observan como sujetos más riesgosos por su condición de madres.

Asimismo, en la hipótesis específica 2, los resultados del p (Rho) de Spearman con un valor positivo significativo de 0,958 , valida la misma, determinando que los factores psicosociales inciden en el modelo de negocio de los emprendimientos de los graduados de la carrera de Marketing de la Universidad Laica "Eloy Alfaro" de Manabí periodo 2015-2019, por lo que el gobierno debería crear una institución o departamento que apoye a la mujer a emprender tanto en el ámbito técnico del emprender como asistencia empresarial, jurídica, tecnológica y tributaria, hasta aspectos psicosociales que ayuden a fortalecer su confianza. $Y$ también es oportuno la puesta en marcha de un departamento en la Universidad Laica "Eloy Alfaro" de Manabí apoye los emprendimientos del alumnado, y en el caso específico de la mujer, se realice a través de capacitaciones que contribuya con su empoderamiento, con la finalidad de que tenga mayor confianza en la ejecución de acciones.

De igual manera, en la hipótesis especifica 3, se valida el supuesto, con un $p$ (Rho) de Spearman positivo significativo de 0,982 , lo que demuestra que los factores sociológicos inciden en los factores socioeconómicos de los emprendimientos de los graduados de la carrera de Marketing de la Universidad Laica "Eloy Alfaro" de Manabí periodo 2015-2019, por lo que el gobierno debería realizar una campaña de cambio de enfoque de cultura desde el hogar, la educación y en los empleos, para que los niños desde temprana edad aprendan que deben formarse integralmente, sin dejarse encasillar en los estereotipos, ni crear perjuicios en otros seres humanos.

Por último, en la hipótesis especifica 4, se valida el supuesto, con un $\mathrm{p}$ (Rho) de Spearman positivo significativo de 0,943 , lo que demuestra que los factores sociológicos inciden en el modelo de negocio de los emprendimientos de los graduados de la carrera de Marketing de la Universidad Laica "Eloy Alfaro" de Manabí periodo 2015-2019, por ello los graduados de marketing deben instaurar el cambio en sus negocios, eliminando las barreras de los estereotipos en los mismos emprendimientos, puesto que, si tienen a su cargo empleados, no encasillar a una mujer o algún hombre en un trabajo determinado, simplemente evaluarlos por su desempeño en las actividades que se les designan.

\section{REFERENCIAS}

Cacouault, M. (2000). Variaciones en los empleos femeninos y masculinos. Las nuevas fronteras de la desigualdad. Hombres y mujeres en el mercado de trabajo., 27-34.

Cejka, M., \& Eagly, A. (1999). Gender-stereotypic images of occupations correspond to the sex segregation of employment. Personality and Social Psychology Bulletin, 25, 413-423.

Defensoría del Pueblo. (2016). Política Institucional de Igualdad de Género 2016-2019. Quito, Ecuador: Ecuador. Defensoría del Pueblo. Obtenido de http://repositorio.dpe.gob.ec/handle/39000/1132.

Eagly, A. H. (1982). Inferred sex differences in status as a determinant of gender stereotypes about social influence. Journal of Personality and Social Psychology, 43(5), 915. Obtenido de https://www.researchgate.net/publication/285179532 Social role theory. 
Eagly, A. H., \& Steffen, V. J. (1984). Gender stereotypes stem from the distribution of women and men into social roles. Journal of Personality, 46(4), 735-754.

Eagly, A. H., \& Wood, W. (1982). Inferred sex differences in status as a determinant of gender stereotypes about social influence. Journal of Personality and Social Psychology, 43(5), 915.

ESPAE, E. d. (2020). Reporte del Global Entrepreneurship Monitor (GEM). Guayaquil: BABSON, KOEF-ISSN 1390-3047. Obtenido de https://www.espae.espol.edu.ec/informe-actualizadogem-ecuador-2019-2020/.

Gálvez, L. (2004). Logros y retos del análisis de género en la historia económica de la empresa. Información Comercial Española(812), 77-89.

GEM. (2019-2020). Global Entrepreneurship Monitor 2019/2020. Ecuador: ESPAE-ISSN No. 13903047. Obtenido de https://www.espae.espol.edu.ec/wpcontent/uploads/documentos/GEM Ecuador 2019.pdf.

Gómez Esteban, C. (2000). ¿Subordinadas o tiranas?: Imágenes de la mujer en puestos de responsabilidad laboral. Conocimiento, Mujer, Poder: Las Mujeres En Los Espcios Del Saber y La Autoridad UIMP, Sevilla.

Hernández Sampieri, D., Fernández Collado, D., \& Baptista Lucio, D. (2014). Metodología de la Investigación (Sexta edición ed.). México D.F., México: McGRAW-HILL-ISBN: 978-1-45622396-0.

Kelley, D., Brush, C. G., Greene, P. G., \& Litovsky, Y. (2011). Global Entrepreneurship Monitor 2010 women's report. Babson Park, MA: Babson College.

Massuh, E. L. (2018). Determinar la incidencia del género como influencia en las actividades socioeconómicas laborables de la comunidad que permitan el desarrollo de un emprendimiento focalizado en la isla trinitaria en la ciudad de Guayaquil. Tesis de Ingenieria. Universidad de Guayaquil, Guayaquil. Obtenido de http://repositorio.ug.edu.ec/handle/redug/36344

Morán Abad, I. E., \& Macías Poveda, E. K. (2016). Incidencia del género en el emprendimiento de microempresas en Petrillo del Cantón Nobol, año 2016. Tesis de Ingenieria. Universidad de Guayaquil, Guayaquil, Obtenido de http://repositorio.ug.edu.ec/bitstream/redug/16423/1/TESIS\%20ROMMORMMORAN1113151 12016\%20-250106022017.pdf.

Poma Carcelén, J., \& Mendoza Munzón, S. (2012). Lenguaje sexista, androcéntrico y estereotipos presentes en la escuela Río Guayas del cantón Pablo Sexto. Tesis de Licenciatura. Universidad de Cuenca, Cuenca. Obtenido de http://dspace.ucuenca.edu.ec/handle/123456789/3011.

Santiesteban Naranjo, E. (2014). Metodología de la Investigación Científica. Las Tunas, Cuba: Editorial Académica Universitaria (Edacun), ISBN: 978-959-7225-03-4. Obtenido de https://elibro.net/en/ereader/udla/151737

Schein, V. E. (1975). Relationships between sex role stereotypes and requisite management characteristics among female managers. requisite management characteristics among female managers., 60(3), 340 . 
Steele, C. (1998). Stereotyping and its threat are real. American Psychologist, 53(6), 680-681

Venegas, M. (2011). Un modelo sociológico para investigar. Revista mexicana de sociología, 73(4), 559-589. Obtenido de http://www.scielo.org.mx/pdf/rms/v73n4/v73n4a1.pdf.

Watson, J., \& Newby, R. (2005). Biological sex, stereotypical sex-roles, and SME owner characteristics. International Journal of Entrepreneurial Behaviour \& Research, 11(2), 129143. 